\title{
Change in neutrophil to lymphocyte ratio during immunotherapy treatment is a non-linear predictor of patient outcomes in advanced cancers
}

\author{
Mingjia $\mathrm{Li}^{1}$ - Daniel Spakowicz ${ }^{2,3} \cdot \mathrm{Jarred} \mathrm{Burkart}^{2} \cdot \mathrm{Sandip} \mathrm{Patel}^{2} \cdot$ Marium Husain $^{2} \cdot \mathrm{Kai} \mathrm{He}^{2} \cdot$ Erin M. Bertino ${ }^{2}$. \\ Peter G. Shields ${ }^{2} \cdot$ David P. Carbone $^{2}$. Claire F. Verschraegen ${ }^{2} \cdot$ Carolyn J. Presley $^{2} \cdot$ Gregory A. Otterson $^{2}$. \\ Kari Kendra² . Dwight H. Owen² ${ }^{2}$
}

Received: 17 June 2019 / Accepted: 16 July 2019 / Published online: 31 July 2019

(c) The Author(s) 2019

\begin{abstract}
Background The neutrophil to lymphocyte ratio (NLR) is known to be prognostic for patients with advanced cancers treated with immune checkpoint inhibitors (ICI), but has generally been evaluated as a single threshold value at baseline. We evaluated NLR at baseline and within first month during treatment in patients who received ICI for advanced cancer to evaluate the prognostic value of baseline and of changes from baseline to on-treatment NLR.

Methods A retrospective review of patients with advanced cancer treated with ICI from 2011 to 2017 at the Ohio State University was performed. NLR was calculated at the initiation of ICI and repeated at median of 21 days. Overall survival (OS) was calculated from the initiation of ICI to date of death or censored at last follow-up. Significance of Cox proportional hazards models were evaluated by log-rank test. Calculations were performed using the survival and survminer packages in $R$, and SPSS.

Results 509 patients were identified and included in the analysis. Patients with baseline and on-treatment NLR $<5$ had significantly longer OS $(P<0.001)$. The change in NLR overtime was a predictor of OS and was observed to be non-linear in nature. This property remained statistically significant with $P<0.05$ after adjusting for age, body mass index, sex, cancer type, performance status, and days to repeat NLR measurement. Patients with a moderate decrease in NLR from baseline had the longest OS of 27.8 months (95\% CI 21.8-33.8). Patients with significant NLR decrease had OS of 11.4 months (95\% CI 6.1-16.7). Patients with a significant increase in NLR had the shortest OS of 5.0 months (95\% CI 0.9-9.1).

Conclusions We confirmed the prognostic value of NLR in patients with advanced cancer treated with ICIs. We found that change in NLR over time is a non-linear predictor of patient outcomes. Patients who had moderate decrease in NLR during treatment with ICI were found to have the longest survival, whereas a significant decrease or increase in NLR was associated with shorter survival. To our knowledge, this is the first study to demonstrate a non-linear change in NLR over time that correlates with survival.
\end{abstract}

Keywords Immunotherapy $\cdot$ Immune checkpoint inhibitor $\cdot$ Prognosis $\cdot$ Neutrophil to lymphocyte ratio $\cdot$ NLR

Dwight H. Owen

Dwight.owen@osumc.edu

1 Division of Hospital Medicine, Department of Internal Medicine, Ohio State University Wexner Medical Center, Columbus, OH, USA

2 Division of Medical Oncology, Department of Internal Medicine, Ohio State University Wexner Medical Center, $320 \mathrm{~W} 10$ th Ave, A450B Starling Loving Hall, Columbus, OH 43210, USA

3 Department of Biomedical Informatics, Ohio State University Wexner Medical Center, Columbus, OH, USA

$\begin{array}{ll}\text { Abbreviations } \\ \text { CBC } & \text { Complete blood count } \\ \text { CTLA-4 } & \text { Cytotoxic T-lymphocyte-associated protein } 4 \\ \text { ECOG } & \text { Eastern cooperative oncology group } \\ \text { ICI } & \text { Immune checkpoint blocker } \\ \text { NLR } & \text { Lymphocyte to neutrophil ratio } \\ \text { OS } & \text { Overall survival } \\ \text { PD-1 } & \text { Programmed cell dealth-1 } \\ \text { PDL-1 } & \text { Program cell death ligand-1 } \\ \text { TMB } & \text { Tumor mutation burden }\end{array}$




\section{Introduction}

Immune checkpoint inhibitors (ICI) targeting cytotoxic T-lymphocyte-associated protein 4 (CTLA-4) and programmed cell dealth-1/program cell death ligand-1 (PD-1/ PDL-1) proteins are able to augment host anti-tumor immune response (Kantarjian et al. 2016; Patel and Minn 2018). Despite the advent of new immunotherapies, there are few clinical prognostic tools available for patients who are offered treatment with ICIs. Previous studies have evaluated PD-L1 expression and tumor mutational burden (TMB) as potential biomarkers. These markers have shown great promise, but there are significant limitations due to cost, assay variability and tumor heterogeneity of PD-L1 expression (McLaughlin et al. 2016; Rimm et al. 2017), need for adequate tissue, requirement for invasive biopsy, and lack of standardization for interpretation of TMB (Rizvi et al. 2015; Carbone et al. 2017; Hellmann et al. 2018).

Cancer-associated inflammation leads to poor survival (Naqash et al. 2018; Hanahan and Weinberg 2011; Mantovani et al. 2008). Neutrophil to lymphocyte ratio (NLR) has been studied as a marker for systemic inflammation (Naqash et al. 2018). NLR has been demonstrated to be prognostic for cancer patients who have received ICI, with low baseline NLR at the start of ICIs being associated with favorable clinical outcomes (Naqash et al. 2018; Ameratunga et al. 2018; Lalani et al. 2018; Sacdalan et al. 2018; Lawati 2018; Park et al. 2018; Bagley et al. 2017; Zaragoza et al. 2015; Khoja et al. 2016). In past studies, the decrease in NLR during treatment was associated with improved survival. However, the decrease in NLR has not been quantitated. Instead, patients with decreasing NLR were combined into a single cohort with favorable OS. To evaluate whether there is any association between the degree of change and overall survival, we studied NLR at baseline and during ICI treatments in patients with advanced cancer at our institution.

\section{Patients and methods}

We identified 509 patients with advanced cancer from 2011 to 2017 at the Ohio State University Comprehensive Cancer Center who received at least 1 dose of ICI as part of a retrospective study approved by the institutional review board at the Ohio State University.

\section{Data collection}

Clinically relevant data were collected in REDCap after retrospective review of electronic medical records (Harris et al. 2009). Patients' baseline complete blood count (CBC) with differential was collected on the day before receiving ICI or within 7 days prior to initiation of ICI. On-treatment CBC was collected at the next blood draw. If on-treatment $\mathrm{CBC}$ was less than 7 days from the initiation of ICI, a CBC closest to 14 days post-initiation of ICI was used. Clinical characteristics were extracted from the medical record including age at ICI, gender, cancer type and stage, performance status at time of ICI, BMI, and type of immunotherapy.

\section{Statistical analysis}

NLR was defined as the absolute neutrophil count divided by the absolute lymphocyte count obtained from CBC. Overall survival (OS) was reported in number of months from the initiation of ICI to the date of death or last follow-up. All univariate and multivariate analyses were conducted using survminer package in R and IMB SPSS Version 25. Univariate analyses used Kaplan-Meier survival curves with log-rank test. Multivariate analyses used Cox proportional hazards models, defining the hazard function for each patient $k$ as:

$\left.h_{\mathrm{k}}(t)=h_{0}(t) e^{\left\{\sum_{t=1}^{n} \beta_{1} N_{\mathrm{B}}+\beta_{2} N_{\mathrm{C}}+\beta_{3} N_{\mathrm{C}}^{2}\right.}\right\}$,

where $h(t)$ is the hazard function at time $t=1$ to $n, N_{\mathrm{B}}$ the baseline NLR value, $N_{\mathrm{C}}$ the change in NLR from baseline to treatment, and $N_{\mathrm{C}} 3$ is the cubic change in NLR. The same method was used to control additional covariates, including BMI, age, sex, time to the second NLR measurement, Eastern Cooperative Oncology Group (ECOG) performance status, cancer, and immunotherapy treatment. Model evaluation, particularly to determine whether a polynomial change in NLR demonstrated significant improvement in the model and which exponentiation, was performed using a likelihood ratio test of nested models, implemented in the lmtest package in $R$ (Kantarjian and Wolff 2016). Code to reproduce all analyses and figures can be found at https://github.com/ spakowiczlab/nlr-io.

\section{Results}

\section{Patient characteristics}

Patient characteristics for the 509 patients are summarized in Table 1. There were 314 (61.7\%) male and 195 (38.3\%) female. The mean age was 62.7 years at the start of ICI, and 254 patients (49.9\%) had melanoma, $111(21.8 \%)$ had non-small cell lung cancer (NSCLC), 68 (13.4\%) had renal cell carcinoma, 47 (9.2\%) had head and neck cancer, 19 (3.7\%) had bladder cancer, and $10(2.0 \%)$ had sarcoma. The majority of patients had metastatic disease, with 69 (13.6\%) patients treated for stage 3 cancer and 427 for stage 4 . ICI 
Table 1 Patient characteristics

\begin{tabular}{llllll}
\hline Total patients & 509 & & & \\
\hline Male & $314(61.7 \%)$ & Baseline NLR & 5.7 (mean) & Time to repeat lab & \\
Female & $195(38.3 \%)$ & Treatment NLR & 6.24 (mean) & Median & 21 days \\
Age & $62.7($ mean) & BMI & 28.86 & & \\
Cancer types & & Stage & & Immunotherapy & \\
NSCLC & $111(21.8 \%)$ & 3 & $69(13.6 \%)$ & Ipilimumab & $163(32.0 \%)$ \\
Melanoma & $254(49.9 \%)$ & 4 & $427(83.9 \%)$ & Nivolumab & $202(39.7 \%)$ \\
Renal cell Ca & $68(13.4 \%)$ & Other & $13(2.5 \%)$ & Pembrolizumab & $88(17.3 \%)$ \\
Head and neck & $47(9.2 \%)$ & & & Nivo + Ipi & $34(6.7 \%)$ \\
Bladder cancer & $19(3.7 \%)$ & & Other & $22(4.2 \%)$ \\
Sarcoma & $10(2.0 \%)$ & & & & \\
\hline
\end{tabular}

Fig. 1 Kaplan-Meier survival analysis for patients with baseline is represented in dotted line and on-treatment in solid line. Patients were grouped based on NLR $<5$ and NLR $\geq 5$. Patients with lower NLR at baseline and on-treatment were associated with better prognosis

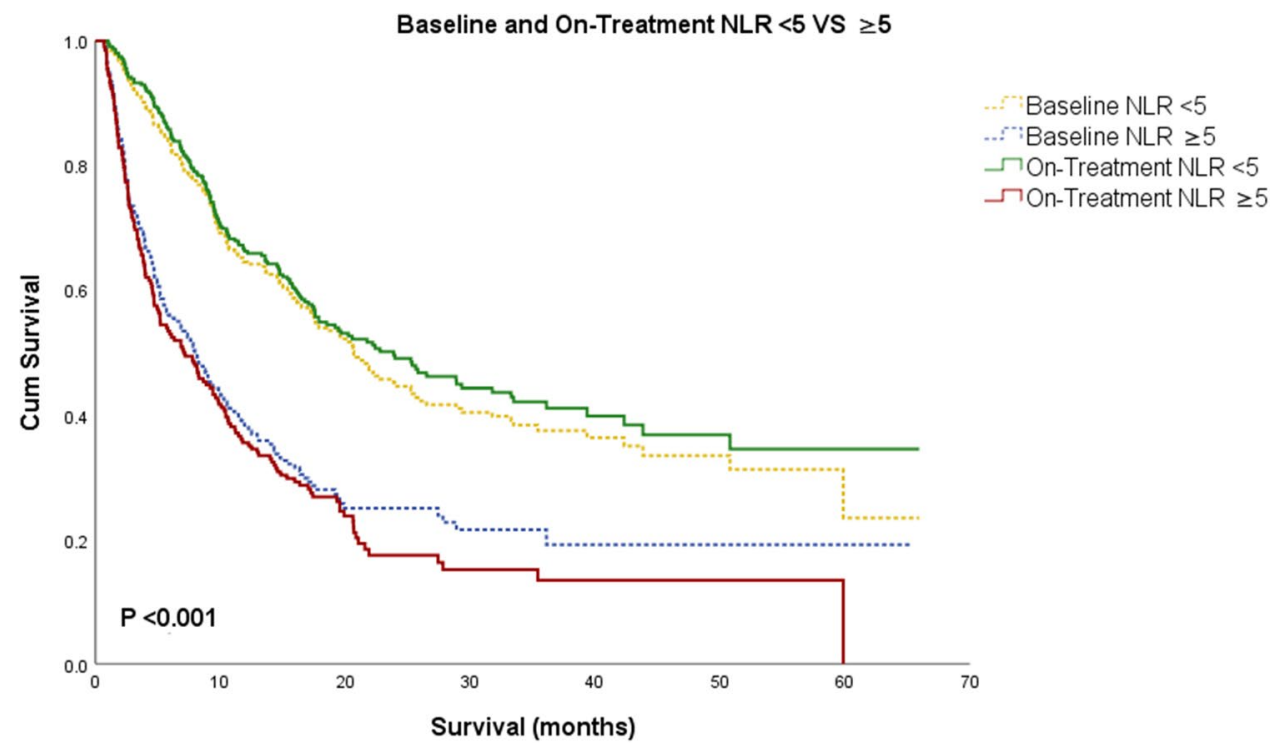

treatment included nivolumab in $202(39.7 \%)$ patients, ipilimumab in 163 (32.0\%), pembrolizumab in 88 (6.7\%), and nivolumab plus ipilimumab combination therapy in 34 (6.7\%), other ICI 22 (4.3\%).

\section{Baseline NLR}

Patients with lower NLR at baseline were associated with improved OS. Patients were separated in two categories of NLR $<5$ and $\geq 5$, as previously done (Ameratunga et al. 2018; Park et al. 2018; Bagley et al. 2017; Capone et al. 2018; Sacdalan et al. 2018). Patients with baseline NLR $<5$ had a median OS of 20.7 months (95\% CI 17.1-24.5) compared to a median OS of 7.9 months (95\% CI 5.8-10.1) in patients with baseline NLR $\geq 5(P<0.001$, Fig. 1).

\section{On-treatment NLR}

On-treatment NLR in our study was also associated with improved overall survival. The median time between baseline and on-treatment NLR was 21 days. Lower on-treatment NLR was associated with a significantly improved OS: 311 patients with on-treatment NLR $<5$ had a median OS of 23.9 months (95\% CI 17.6-30.1), whereas 198 patients with on-treatment NLR $\geq 5$ had a median OS of 7.1 months $(95 \%$ CI 4.7-9.4, $P<0.001$, Fig. 1).

\section{Change in NLR during treatment}

Following the observation that on-treatment NLR better stratified patient outcomes, we tested the hypothesis that the value of NLR was less important than its trend. Specifically, a starting NLR $\geq 5$ was less important than whether it decreased on ICI treatment. The change in NLR was observed as a univariate Kaplan-Meier survival plot. A maximally selected rank test showed that the optimal stratum for the change in NLR was at 0.9, and indeed this value strongly stratified overall survival of patients $(P<0.001$, Fig. 2a). 
Fig. 2 The change in NLR from baseline to on-immunotherapy measurements shows curvilinear character. a Survival curve stratifying by change in NLR of greater than 0.9. b Loesssmoothed fit of the change in NLR and months to last followup or death across several cancer types. b Cox proportional hazards model showing a significant polynomial term describing the change in NLR. The cubic term yielded a lower log likelihood than the quadratic change in NLR and the quadratic term did not significantly improve the model in addition to the cubic term
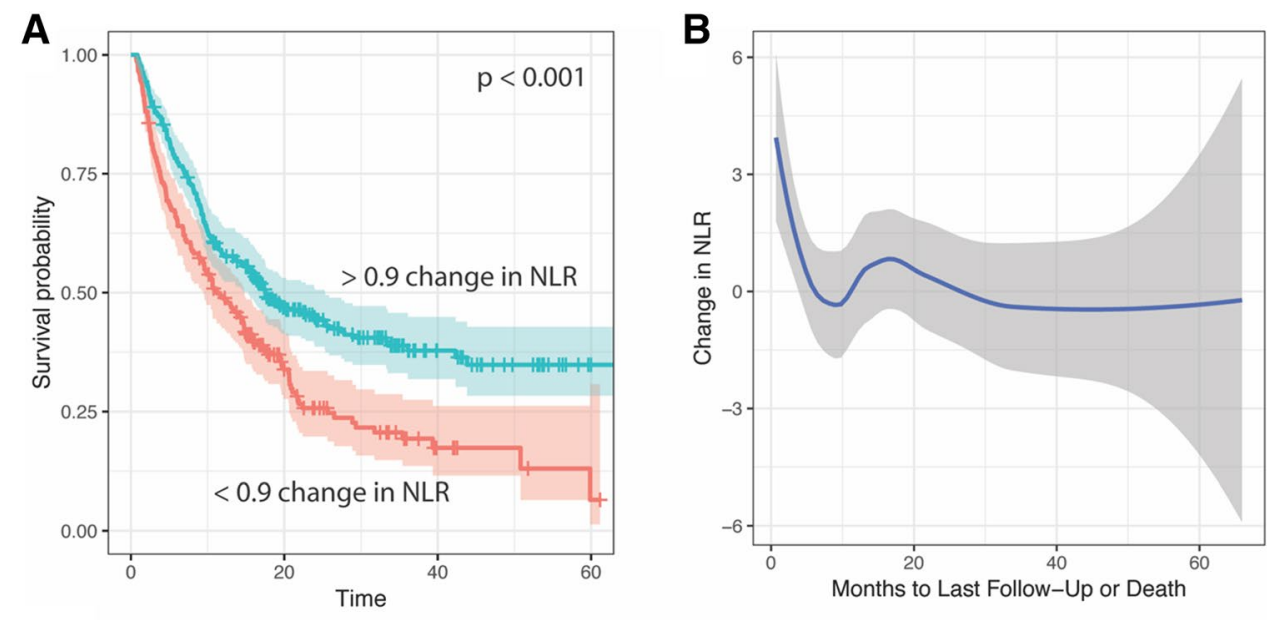

C

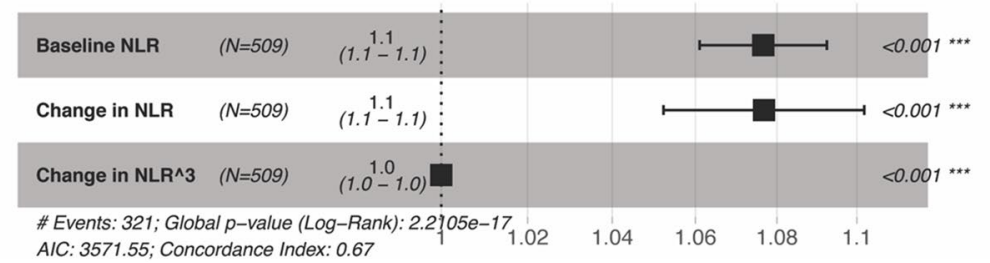

To rule out dependence on when the on-treatment NLR was measured (that is, the change in NLR could be gradual, and patients who were tested relatively early after the baseline NLR may show less change), we, therefore, visualized the change in NLR based on the time of the repeat test, and observed no relationship (slope not significantly different from 0 , Fig. 2b). However, we noticed that the changes in NLR were not linearly related to survival. A loess fit showed strong curves at times of death $<12$ months (Fig. 2c).

To evaluate whether the non-linear change in NLR significantly improves the accuracy of survival models, we pursued a multivariate Cox proportional hazards approach. In a model for overall survival that included the baseline NLR value, the change in NLR was also a significant predictor, as suggested by the survival plot (Fig. 2a). We explored this relationship further by observing the change in NLR with respect to the number of months to last follow-up or death (Fig. 2b). This showed strongly curvilinear features, where highly positive changes in NLR predict poor outcomes, and those with the longest survival showed a decrease in NLR of less than one. However, the addition of a polynomial on the change in NLR was also significant. A series of polynomials on the change in NLR were tested by likelihood ratio test of nested models, which demonstrated that a cubic term most improved the model (Fig. 2c). This cubic term remained a significant predictor when controlling for age, BMI, sex, ECOG performance status, cancer, and the type of immunotherapy received. NLR is, therefore, the best fit to outcome non-linearly, in the sense that the relationship is not a straight line, but rather a curvilinear relationship or polynomial linear function.
This effect was apparent when we stratified the cohort by their change in NLR. Using semi-arbitrary strata, 45 patients showed a large increase in NLR by more than $100 \%, 311$ patients changed little (as defined by increase less than $100 \%$ to decrease less than $16.7 \%$ ), 53 patients decreased moderately (16.7\%-28.5\%), and 100 patients showed a large decrease (beyond 28.5). Patients with a moderate decrease in NLR (between 16.7\% and 28.5\%) had the longest OS with median OS of 27.8 months (95 CI 21.8-33.8), whereas patients with decrease in NLR greater than $28.5 \%$ had OS of 11.4 months (95 CI 6.1-16.7) months. Those who had significant increase in NLR by more than $100 \%$ during treatment had the shortest OS at 5.03 (95 CI 0.94-9.1) months (overall $P<0.001$, Fig. 3).

\section{Discussion}

In the era of cancer immunotherapy, indications for ICI use in cancer care are expanding at a rapid pace (Antonia et al. 2017; Marin-Acevedo et al. 2018). Previous studies explored high PDL-1 expression and tumor mutation burden (TMB) as potential predictive biomarkers. Patients with high tumor expression of PD-L1 tend to have a better outcome (Garon et al. 2015; Gettinger et al. 2014; Reck et al. 2016). However, the durable benefit seen in some patients without PD-L1 expression limits its role as an exclusionary prognostic biomarker. TMB has also shown promise (Rizvi et al. 2015; Carbone et al. 2017; Hellmann et al. 2018). However, there is a lack of standardization for TMB calculation, a variety of assays available, and not all tumor mutations will 
Fig. 3 Kaplan-Meier survival analysis showing change in NLR from baseline to on-treatment based on the percentage of change. Patient with modest decrease in NLR had the longest median OS

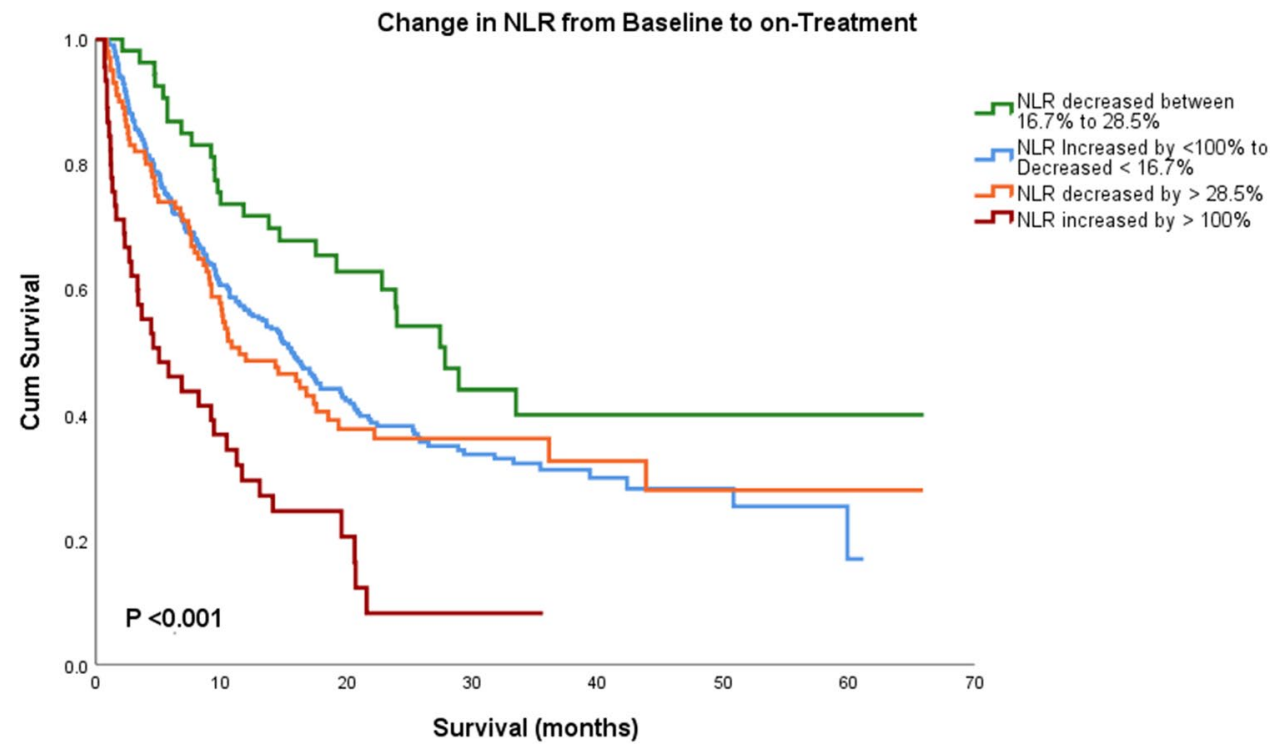

lead to altered proteins that are measured by TMB (Rizvi et al. 2015; Carbone et al. 2017). Furthermore, both PDL-1 expression and TMB analysis require expensive and invasive biopsies to obtain tissue samples, which are impractical to use for frequent biomarker monitoring. Recent evidence has suggested the microbiome as an additional marker, though it has not been widely adopted (Routy et al. 2018).

In this study, we confirmed the prognostic value of NLR at the initiation of ICI treatment. Interestingly, the relationship between NLR and survival was non-linear. Only a moderate decrease in NLR was associated with the longest OS. An excessive decline in NLR was associated with reduced OS.

NLR is calculated from existing routine labs for patients who are receiving ICI and it is obtained with a routine peripheral blood draw. The inexpensive and readily available nature of NLR allows it to be conveniently monitored overtime. The change in NLR is a useful biomarker to the extent that it can be calculated in a timely manner in the clinic. Ideally, the baseline NLR value would stratify patients who will respond to ICI vs. those who are better suited to alternative treatments such as chemotherapy. More study is needed to validate this prediction, and if it holds, to identify the optimal time for repeat NLR.

The mechanism by which NLR relates to ICI activity and OS is unknown. Our results show that large increases or decreases are negatively associated with OS. Large increases in NLR may reflect increased tumor burden, lack of ICI efficacy and, therefore, decreased OS. Large decreases in NLR are more challenging to interpret. We observed that change in NLR is driven primarily by the decline in neutrophils and that the lymphocyte count remained relatively constant in our patient population. The common causes of decline in neutrophils can include decreased bone marrow activity, infection, and malnutrition, all of which have been shown to impact OS (Bouteloup et al. 2017). Agranulocytosis and neutropenia may also rarely occur as a result of auto-immune toxicity from ICI treatment (Barbacki et al. 2018).

This is the first study to demonstrate the non-linear relationship between change in NLR and OS during ICI treatment. There are several limitations in our study, including its retrospective nature, unknown mechanism of action, inclusion of a heterogeneous patient population, and the inability to evaluate variables with known predictive power in this context, notably the line of therapy and mutation status. Further studies looking into relationship between NLR and tumor micro-environment, medication interactions, infection, nutrition status, microbiota, line of therapy, and immunerelated adverse events may help to delineate the mechanisms between the non-linear change in NLR and OS. Prospective studies with a larger patient cohort are needed for validation.

\section{Conclusion}

This is the first study to demonstrate the non-linear relationship between change in NLR and survival during ICI treatment for patients with advanced cancer. Further studies looking into the reasons for this non-linear relationship, including possibly the contributions of tumor micro-environment, infection, nutrition status, microbiota, and other immune-related adverse events to change in NLR may help to delineate the mechanisms between the non-linear change in NLR and clinical outcomes.

\section{Acknowledgements None.}

Author contributions Conception and design: Mingiia Li, Daniel Spakowicz, Gregory A. Otterson, Kari Kendra, Dwight H. Owen. Data 
acquisition and analysis: Mingjia Li, Daniel Spakowicz, Jarred Burkart, Sandip Patel, Marium Husain, Kai He, Carolyn J. Presley, Erin M. Bertino, Peter G. Shields, David P. Carbone, Claire F. Verschraegen, Gregory A. Otterson, Kari Kendra, Dwight H. Owen. Data interpretation: Mingjia Li, Daniel Spakowicz, David P. Carbone, Claire F. Verschraegen, Gregory A. Otterson, Kari Kendra, Dwight H. Owen. Drafting/substantial revisions: Mingjia Li, Daniel Spakowicz, Carolyn J. Presley, Erin M. Bertino, Peter G. Shields, David P. Carbone, Claire F. Verschraegen, Gregory A. Otterson, Kari Kendra, Dwight H. Owen. Approval of final version: Mingiia Li, Daniel Spakowicz, Jarred Burkart, Sandip Patel, Marium Husain, Kai He, Carolyn J. Presley, Erin M. Bertino, Peter G. Shields, David P. Carbone, Claire F. Verschraegen, Gregory A. Otterson, Kari Kendra, Dwight H. Owen.

Funding This project utilized REDCap database software which was supported by The Ohio State University Center for Clinical and Translational Science grant support (National Center for Advancing Translational Sciences, Grant 8UL1TR000090-05).

Data availability The datasets used and/or analyzed during the current study are available from the corresponding author on reasonable request.

\section{Compliance with ethical standards}

Conflict of interest The authors declare that they have no conflict of interest.

Ethics approval Approved by IRB at the Ohio State University (\#2016C0070).

Informed consent Not applicable.

Open Access This article is distributed under the terms of the Creative Commons Attribution 4.0 International License (http://creativeco mmons.org/licenses/by/4.0/), which permits unrestricted use, distribution, and reproduction in any medium, provided you give appropriate credit to the original author(s) and the source, provide a link to the Creative Commons license, and indicate if changes were made.

\section{References}

Ameratunga $\mathrm{M}$ et al (2018) Neutrophil-lymphocyte ratio kinetics in patients with advanced solid tumours on phase I trials of PD-1/ PD-L1 inhibitors. Eur J Cancer 89:56-63

Antonia SJ et al (2017) Durvalumab after chemoradiotherapy in stage III non-small-cell lung cancer. New Engl J Med 377(20):1919-1929

Bagley SJ et al (2017) Pretreatment neutrophil-to-lymphocyte ratio as a marker of outcomes in nivolumab-treated patients with advanced non-small-cell lung cancer. Lung Cancer 106:1-7

Barbacki A et al (2018) A case of severe Pembrolizumab-induced neutropenia. Anticancer Drugs 29(8):817-819

Bouteloup M, Perinel S, Bourmaud A, Azoulay E, Mokart D, Darmon M, pour le Groupe de Recherche en Reanimation Respiratoire du patient dO-H (2017) Outcomes in adult critically ill cancer patients with and without neutropenia: a systematic review and meta-analysis of the Groupe de Recherche en Reanimation Respiratoire du patient d'Onco-Hematologie (GRRR-OH). Oncotarget 8:1860-1870

Capone $\mathrm{M}$ et al (2018) Baseline neutrophil-to-lymphocyte ratio (NLR) and derived NLR could predict overall survival in patients with advanced melanoma treated with nivolumab. J Immuno Ther Cancer 6(1):74

Carbone DP et al (2017) First-line nivolumab in stage IV or recurrent non-small-cell lung cancer. N Engl J Med 376(25):2415-2426
Garon EB et al (2015) Pembrolizumab for the treatment of non-small-cell lung cancer. N Engl J Med 372(21):2018-2028

Gettinger SN, Shepherd FA, Antonia SJ et al (2014) First-line nivolumab (anti-PD-1; BMS-936558, ONO-4538) monotherapy in advanced NSCLC: Safety, efficacy, and correlation of outcomes with PD-L1 status. J Clin Oncol 32:5s

Hanahan D, Weinberg RA (2011) Hallmarks of cancer: the next generation. Cell 144(5):646-674

Harris PA et al (2009) Research electronic data capture (REDCap)-a metadata-driven methodology and workflow process for providing translational research informatics support. J Biomed Inform 42(2):377-381

Hellmann MD et al (2018) Nivolumab plus Ipilimumab in lung cancer with a high tumor mutational burden. N Engl J Med 378(22):2093-2104

Kantarjian H, Wolff RA, and University of Texas M.D. Anderson Cancer Center (2016) The MD Anderson manual of medical oncology. McGraw-Hill Education, New York, p. xxvii, 1251 pages

Khoja L et al (2016) The full blood count as a biomarker of outcome and toxicity in ipilimumab-treated cutaneous metastatic melanoma. Cancer Med 5(10):2792-2799

Lalani AA et al (2018) Change in neutrophil-to-lymphocyte ratio (NLR) in response to immune checkpoint blockade for metastatic renal cell carcinoma. J Immunother Cancer 6(1):5

Lawati YA et al (2018) The prognostic role of neutrophil-to-lymphocyte ratio trends during therapy in esophageal cancer. 36(4_suppl): 17-17

Mantovani A et al (2008) Cancer-related inflammation. Nature 454:436

Marin-Acevedo JA et al (2018) Next generation of immune checkpoint therapy in cancer: new developments and challenges. J Hematol Oncol 11(1):39

McLaughlin J et al (2016) Quantitative assessment of the heterogeneity of PD-L1 expression in non-small-cell lung cancer. JAMA Oncol 2(1):46-54

Naqash AR et al (2018) Co-relation of overall survival with peripheral blood-based inflammatory biomarkers in advanced stage nonsmall cell lung cancer treated with anti-programmed cell death-1 therapy: results from a single institutional database. Acta Oncol 57(6):867-872

Park W et al (2018) Developing a predictive model for clinical outcomes of advanced non-small cell lung cancer patients treated with nivolumab. Clin Lung Cancer 19(3):280-288

Patel SA, Minn AJ (2018) Combination cancer therapy with immune checkpoint blockade: mechanisms and strategies. Immunity 48(3):417-433

Reck M et al (2016) Pembrolizumab versus chemotherapy for PD-L1-positive non-small-cell lung cancer. N Engl J Med 375:1823-1833

Rimm DL et al (2017) A prospective, multi-institutional, pathologist-based assessment of 4 immunohistochemistry assays for PD-L1 expression in non-small cell lung cancer. JAMA Oncol 3(8):1051-1058

Rizvi NA et al (2015) Cancer immunology mutational landscape determines sensitivity to PD-1 blockade in non-small cell lung cancer. Science 348(6230):124-128

Routy B et al (2018) Gut microbiome influences efficacy of PD-1-based immunotherapy against epithelial tumors. Science 359(6371):91-97

Sacdalan DB, Lucero JA, Sacdalan DL (2018) Prognostic utility of baseline neutrophil-to-lymphocyte ratio in patients receiving immune checkpoint inhibitors: a review and meta-analysis. Onco Targets Ther 11:955-965

Zaragoza J et al (2015) High neutrophil to lymphocyte ratio measured before starting ipilimumab treatment is associated with reduced overall survival in patients with melanoma. Br J Dermatol 174(1):146-151

Publisher's Note Springer Nature remains neutral with regard to jurisdictional claims in published maps and institutional affiliations. 